\title{
miRNA-21 enhances chemoresistance to cisplatin in epithelial ovarian cancer by negatively regulating PTEN
}

\author{
XIAOMIN YU ${ }^{1 *}$, YULONG CHEN $^{2 *}$, RUIYUN TIAN $^{1}$, JIANXIA LI $^{1}$, HONGYAN LI $^{1}$, TENG LV $^{2}$ and QIN YAO $^{2}$ \\ ${ }^{1}$ Department of Obstetrics and Gynecology, The Eighth People's Hospital of Qingdao, Qingdao, Shandong 266100; \\ ${ }^{2}$ Department of Obstetrics and Gynecology, Affiliated Hospital of Qingdao University, Qingdao, Shandong 266003, P.R. China
}

Received September 26, 2015; Accepted February 16, 2017

DOI: $10.3892 / \mathrm{ol} .2017 .6324$

\begin{abstract}
MicroRNAs (miRNAs) are small non-coding RNAs, 8-23 nucleotides in length, which regulate gene expression at the post-transcriptional level. The present study was performed to analyze the association between microRNA-21 and cisplatin resistance in epithelial ovarian cancer (EOC) SKOV3 and SKOV3/DDP cells. In this experiment, the resistance of SKOV3 and SKOV3/DDP cells to cisplatin was evaluated using the MTT assay. Reverse transcription-quantitative polymerase chain reaction analysis was used to assess miRNA-21 levels and phosphatase and tensin homolog (PTEN) mRNA levels. Western blotting was used to assess PTEN protein levels. miRNA-21 mimics or inhibitors were transfected into SKOV3 and SKOV3/DDP cells. Prior to transfection, higher expression levels of miRNA-21 were observed in SKOV3/DDP cells compared with SKOV3 cells. Following transfection with miRNA-21 mimics, SKOV3 cells demonstrated increased sensitivity to cisplatin compared with negative control cells. Following transfection with the miRNA-21 inhibitor, SKOV3/DDP cells demonstrated decreased sensitivity to cisplatin compared with negative control cells. Furthermore, PTEN mRNA expression levels in SKOV3 cells transfected with miRNA-21 mimics was significantly lower compared with negative control cells. These results suggested that miRNA-21 may regulate cisplatin resistance by negatively targeting PTEN in EOC.
\end{abstract}

\section{Introduction}

Epithelial ovarian cancer (EOC) is one of the most common malignant gynecologic tumors. Debulking surgery followed by

Correspondence to: Professor Qin Yao, Department of Obstetrics and Gynecology, Affiliated Hospital of Qingdao University, 16 Jiangsu Road, Qingdao, Shandong 266003, P.R. China

E-mail: dr_yaoqin@163.com

*Contributed equally

Key words: microRNA-21, Epithelial ovarian cancer, cisplatin, drug-resistance, PTEN a combination of platinum and taxane based chemotherapy are widely used treatments for EOC at present. Although overall survival rates have increased slightly over the past 25 years, 5 -year survival remains $<50 \%$ (1). The high mortality rate of ovarian cancer is due to late-stage diagnosis and resistance to platinum-based chemotherapy. However, the mechanisms underlying cisplatin resistance in EOC remain to be fully understood.

MicroRNAs (miRNAs) are small non-coding RNAs of 8-23 nucleotides that post-transcriptionally regulate gene expression. Multiple previous reports have indicated that dysregulation of miRNA target genes promotes drug resistance, and inhibition of miRNAs may reverse drug resistance $(2,3)$. miRNA-21 is overexpressed in multiple types of cancer, and promotes the initiation of cancer, progression and drug-resistance (4-9). miRNA-21 impacts tumorigenesis by negatively regulating several targets. Phosphatase and tensin homolog (PTEN) is a tumor suppressor molecule. Inactivating mutations and deletions of the PTEN gene have been observed in multiple types of cancer. Notably, bioinformatics tools have demonstrated that the 3'-untranslated region of the PTEN gene harbors a putative binding site for miRNA-21 (10). miRNA-21 expression has been revealed to be markedly increased in ovarian cancer compared with benign ovarian tumor tissues (11). miRNA-21 expression was also demonstrated to be increased in drug-resistant ovarian cancer compared with drug-sensitive ovarian cancer serum. In the present study, miRNA-21 mimics, inhibitors and negative control were transfected in to SKOV3 or SKOV3/DDP cells. The PTEN gene was hypothesized to be regulated by miRNA-21 in ovarian cancer cisplatin resistance.

\section{Materials and methods}

Cell lines and cell culture. The SKOV3 human ovarian cancer cell line was purchased from Shanghai Institutes for Biological Sciences, Chinese Academy of Sciences (Shanghai, China). The SKOV3/DDP human ovarian cancer cell line was purchased from the Affiliated Hospital of Qingdao University (Qingdao, China). The cells were cultured in RPMI-1640 medium supplemented with $10 \%$ fetal bovine serum (both from Gibco; Thermo Fisher Scientific, Inc., Waltham, MA, USA), $100 \mathrm{U} / \mathrm{ml}$ penicillin and $100 \mu \mathrm{g} / \mathrm{ml}$ streptomycin, in a humidified cell incubator with an atmosphere of $5 \% \mathrm{CO}_{2}$ and a temperature of $37^{\circ} \mathrm{C}$. Cells from the exponential growth phase 
were used for the following experiments. The treatment groups of cells were as follows: SKOV3/DDP group; SKOV3 group; the negative control group (no drug); the blank control group (no cells). Cells were cultured for $24 \mathrm{~h}$ in a humidified cell incubator with an atmosphere of $5 \% \mathrm{CO}_{2}$ and a temperature of $37^{\circ} \mathrm{C}$.

A total of $200 \mu 1$ of $4 \times 10^{4}$ cells/ml SKOV3/DDP, SKOV3 or cell-free medium were seeded in a 96-well plate. Cisplatin (Qilu Pharmaceutical Co., Ltd., Jinan, China) to a concentration of $0,3.125,6.25,12.5,25,50,100$ or $200 \mu \mathrm{mol} / 1$ was added to each well. The cells were incubated in a humidified cell incubator with an atmosphere of $5 \% \mathrm{CO}_{2}$ and a temperature of $37^{\circ} \mathrm{C}$ for $48 \mathrm{~h}$. A total of $20 \mu \mathrm{l} \mathrm{MTT}$ was added to each well with a concentration of $5 \mathrm{mg} / \mathrm{ml}$. Following a 4-h incubation at $37^{\circ} \mathrm{C}$, the supernatant was discarded using pipettes. A total of $150 \mu \mathrm{l}$ DMSO was added to each well.

A microplate reader was used to analyze the absorbance of each well at an optical density of $490 \mathrm{~nm}$. The cells inhibitory rate (IR) $(\%)=[(1-($ group value-blank control group value)/(negative control group value-blank control group value)]x100\%. Subsequently, the half-maximal inhibitory concentration (IC50) was determined. Resistant factor $(\mathrm{RF})=\mathrm{IC} 50$ of SKOV3 DDP/IC50 of SKOV3 (12).

RNA isolation. Total RNA was extracted and isolated from $2 \times 10^{5}$ cells $/ \mathrm{ml}$ using the mirVana miRNA isolation kit (Ambion; Thermo Fisher Scientific, Inc., Waltham, MA, USA). The quality and quantity of the RNA samples were assessed by standard electrophoresis and spectrophotometric methods (260/280 absorbance).

Transfection. The miRNA-21 mimics and miRNA-21 inhibitor were purchased from Shanghai GenePharma Co., Ltd (Shanghai, China). SKOV3 and SKOV3/DDP cells $\left(2 \times 10^{5}\right.$ cells $\left./ \mathrm{ml}\right)$ were counted and seeded onto 6-well plates the day prior to transfection to ensure $50 \%$ cell confluence on the day of transfection. Transfection of $10 \mathrm{nM}$ miRNA-21 mimics into SKOV3 and transfection of $10 \mathrm{nM}$ miRNA-21 inhibitors, diluted in medium, into SKOV3/DDP cells were performed using Lipofectamine 2000 (Invitrogen; Thermo Fisher Scientific, Inc.) in accordance with the manufacturer's protocol. Lipofectamine was used alone as a negative control. The miRNA-21 mimics/inhibitors were used at a final concentration of $100 \mathrm{nM}$. Reverse transcription-quantitative polymerase chain reaction (RT-qPCR) and western blot analysis were performed $48 \mathrm{~h}$ following transfection.

RT-qPCR analysis. miRNA-21 and PTEN mRNA expression levels were detected by stem-loop RT-qPCR.RT was performed using SYBR Premix Ex Taq (Takara Bio, Inc., Otsu, Japan) at $16^{\circ} \mathrm{C}$ for $30 \mathrm{~min}, 42^{\circ} \mathrm{C}$ for $30 \mathrm{~min}$ and $85^{\circ} \mathrm{C}$ for $5 \mathrm{~min}$. qPCR was performed using SYBR Premix Ex Taq (Takara Bio, Inc.) according to the manufacturer's protocol, with $1.33 \mu \mathrm{l}$ cDNA from the RT reaction. The thermocycler conditions were, an initial $15 \mathrm{~min}$ at $95^{\circ} \mathrm{C}$, then 40 cycles of $15 \mathrm{sec}$ at $95^{\circ} \mathrm{C}$ and $60 \mathrm{sec}$ at $60^{\circ} \mathrm{C}$. The primers for miRNA-21 were as follows: Stem-loop RT, 5'-GTCGTATCCAGTGCAGGGTCCGAGG TATTCGCACTGGATACGACTCAACA-3'; forward, 5'GCC CGCTAGCTTATCAGACTGATG-3' and reverse, 5'-GTG CAGGGTCCGAGGT-3'. The primers for U6 were as follows:
Stem loop RT, 5'-GTCGTATCCAGTGCAGGGTCCGAG GTATTCGCACTGGATACGACAAAAATATG-3'; forward, 5'-GCGCGTCGTGAAGCGTTC-3' and reverse, 5'-GTGCAG GGTCCGAGGT-3'. GAPDH was used to normalize PTEN mRNA expression levels. The forward and reverse primer sequences for PTEN mRNA were as follows: 5'-GAGGGA TAA AACACCATG-3' and 5'-AGGGGTAGGATGTGA ACCAGTA-3', respectively. The forward and reverse primer sequences for GAPDH were 5'-AACTTTGGCATTGTGGAA GG-3' and 5'-ACACATTGGGGGTAGGAACA-3', respectively. All the experiments were performed in triplicate. The relative expression ratios of miRNA-21 and PTEN mRNA in SKOV3 and SKOV3/DDP cell lines was calculated using the $2^{-\Delta \Delta \mathrm{Cq}}$ method (13).

Western blot analysis. A total of $4 \times 10^{4}$ cultured cells were lysed using RIPA buffer (Pierce; Thermo Fisher Scientific, Inc.) in the presence of a Protease Inhibitor Cocktail (Pierce; Thermo Fisher Scientific, Inc.). The protein concentration of the lysates was measured using a BCA Protein Assay kit (Pierce; Thermo Fisher Scientific, Inc.). Equivalent amounts of protein $(0.5 \mathrm{mg} / \mathrm{ml})$ were resolved and mixed with $5 \mathrm{X}$ Lane Marker Reducing Sample Buffer (Pierce; Thermo Fisher Scientific, Inc.), electrophoresed on $12.5 \%$ SDS-acrylamide gel, and transferred to Immobilon-P transfer membranes (Merck KGaA, Darmstadt, Germany). The membranes were blocked with $5 \%$ non-fat milk in Tris-buffered saline at $4^{\circ} \mathrm{C}$ overnight and then incubated with a rabbit anti-human PTEN monoclonal antibody (cat. no., ab32199; dilution, 1:400; Abcam, Cambridge, UK) at $4{ }^{\circ} \mathrm{C}$ overnight followed by horseradish peroxidase (HRP)-conjugated secondary antibody (cat. no., ab151499; dilution, 1:100; Abcam) at room temperature for $1 \mathrm{~h}$. Signals were detected using Immobilon western chemiluminescent HRP substrate (Merck KGaA). $\beta$-actin (cat. no., ab8227; dilution, 1:100; Abcam) served as the loading control.

Statistical analysis. Independent and paired t-tests were used to compare the data. All analyses were performed using SPSS19.0 software (IBM SPSS, Armonk, NY, USA) and all tests were two-tailed. $\mathrm{P}<0.05$ was considered to indicate a statistically significant difference.

\section{Results}

miRNA-21 regulates the sensitivity of SKOV3 and SKOV3/DDP cells to cisplatin. The RT-qPCR results revealed that SKOV3/DDP cells had a higher endogenous miRNA-21 expression level than SKOV3 cells $(\mathrm{P}<0.05$; Fig. 1). The $\mathrm{IC}_{50}$ of control SKOV3 cells was $5.205 \mu \mathrm{mol} / 1$ cisplatin, of SKOV3 cells with the miRNA-21 mimic, $25.763 \mu \mathrm{mol} / 1$, of control SKOV3/DDP cells, $21.914 \mu \mathrm{mol} / 1$, and of SKOV3/DDP cells with the inhibitor, $15.524 \mu \mathrm{mol} / \mathrm{l}$. Thus, the relative cisplatin resistance index of SKOV3 cells transfected with the miRNA-21 mimic was 4.9 compared to the control cells, of the SKOV3/DDP cells compared with SKOV3/DDP cells with the inhibitor, 0.7, and of the control SKOV3/DDP cells relative to the control SKOV3 cells, 4.2. The sensitivity of SKOV3 cells transfected with miRNA-21 mimics to cisplatin was significantly increased compared with the negative 


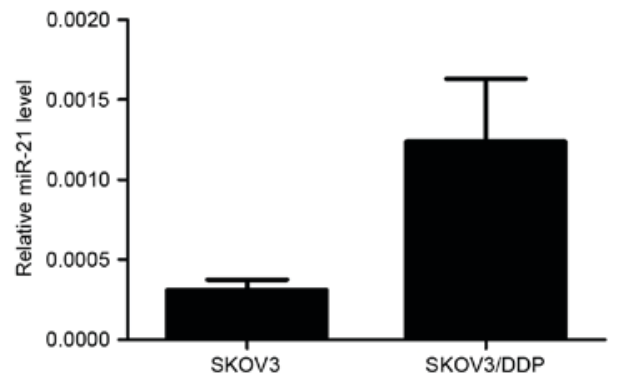

Figure 1. Reverse transcription-quantitative polymerase chain reaction analysis revealed that SKOV3/DDP cells had higher endogenous miRNA-21 expression levels than SKOV3 cells. miRNA, microRNA.

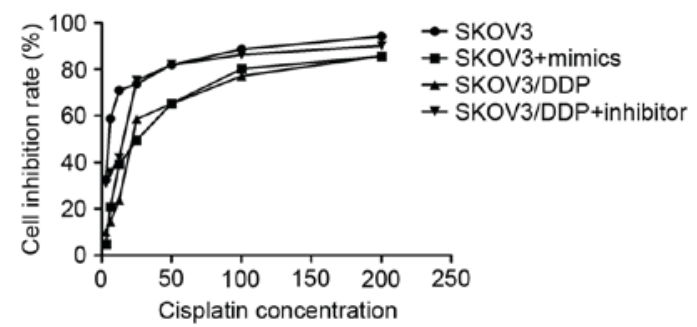

Figure 2. Results of the MTT cell viability assay. Resistance of SKOV3 cells transfected with miRNA-21 mimics to cisplatin was increased compared with negative control cells. SKOV3/DDP cells transfected with miRNA-21 inhibitors demonstrated decreased resistance to cisplatin compared with negative control cells. miRNA, microRNA.

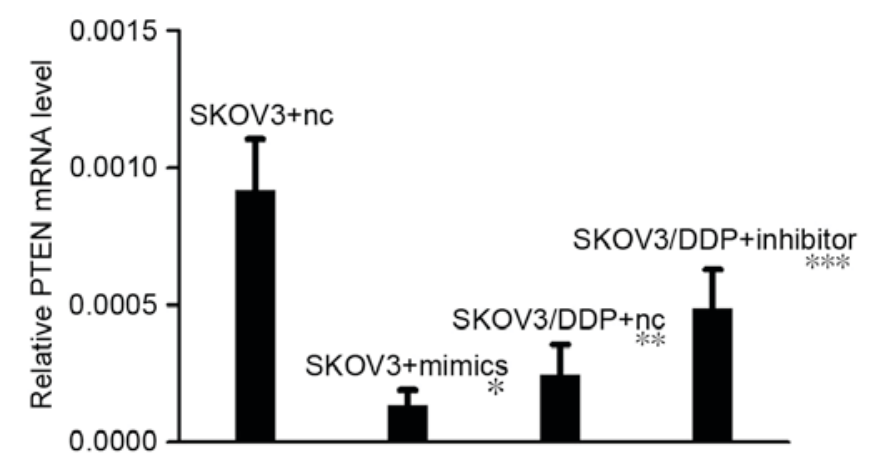

Figure 3. PTEN mRNA expression levels in SKOV3 and SKOV3/DPP cells following transfection with miR-21 mimics and inhibitors, as assessed by reverse transcription-quantitative polymerase chain reaction. A two

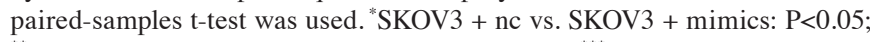
${ }^{* *}$ SKOV3 + nc vs. SKOV3/DDP + nc: P>0.05; ${ }^{* * *}$ SKOV3/DDP + nc vs. SKOV3/DDP + Inhibitor: P $>0.05$. PTEN, phosphatase and tensin homolog; nc, negative control.

control cells $(\mathrm{P}<0.05$; Fig. 2). In addition, SKOV3/DDP cells transfected with miRNA-21 inhibitors were significantly less resistant to cisplatin compared with that of the negative control cells (19.0; $\mathrm{P}<0.05$; Fig. 2).

miRNA-21 downregulates expression of PTEN $m R N A$ and protein in SKOV3 cells. RT-qPCR revealed that, following transfection with miRNA-21 mimics, PTEN mRNA expression levels in the SKOV3 cell line were decreased compared with negative control cells $(\mathrm{P}<0.05$; Fig. 3$)$. When the SKOV3/DDP cell line was transfected with miR-21 inhibitors,

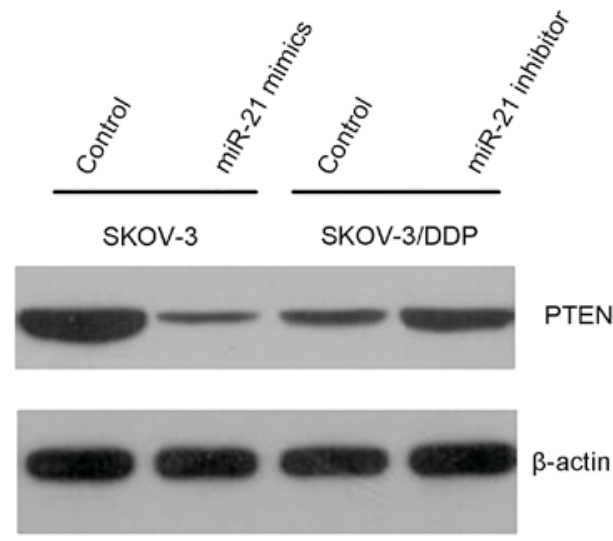

Figure 4. PTEN protein expression levels in SKOV3 and SKOV3/DPP cells following transfection with miR-21 mimics and inhibitors, as assessed by western blotting. PTEN, phosphatase and tensin homolog; miR, microRNA.

PTEN mRNA expression levels were not significantly altered. When miR-21 was transfected into SKOV3 cells, western blot analysis revealed that PTEN protein levels were visibly lower. It was also demonstrated that when miR-21 was transfected into SKOV3/DPP cells line, PTEN protein levels were visibly increased (Fig. 4.).

\section{Discussion}

In the present study, the drug resistance index of SKOV3/DDP cells relative to SKOV3 cells was demonstrated to be 4.2 using the MTT assay. Thus, the present study selected the appropriate cells for experimental study. Although platinum-based chemotherapy has improved the prognosis of ovarian cancer, drug-resistance remains the main obstacle to successful treatment. The present study revealed that multiple miRNAs participate in ovarian cancer drug-resistance, including miRNA-130a and miRNA-374a (14). miRNA-152 and miRNA-185 were demonstrated to be significantly downregulated in SKOV3/DDP and A2780/DDP cells (14). Previous studies have demonstrated that miRNA-21 is involved in drug resistance in multiple types of cancer, including gastric, breast and lung cancer, via regulation of PTEN $(7,8,15)$. In the present study, the expression level of miRNA-21 in SKOV3/DDP cells was significantly higher than in SKOV3 cells, as detected by qPCR. It was demonstrated that miR-21 may participate in ovarian cancer drug-resistance. In the present study, miRNA-21 mimics were transfected into SKOV3 cells, and miRNA-21 inhibitors were transfected into SKOV3/DDP cells. Following transfection, the upregulation of miRNA-21 in SKOV3 cells transfected with the miRNA-21 mimics resulted in an increased cisplatin drug resistance. The downregulation of miRNA-21 in SKOV3/DDP cells transfected with the miRNA-21 inhibitor resulted in decreased cisplatin drug resistance. Thus, the present study further demonstrated miRNA-21 participated in cisplatin resistance in EOC. PTEN mRNA expression levels in SKOV3 cells transfected with the miRNA-21 mimics was significantly decreased compared with negative control cells (cells treated with Lipofectamine alone). However, PTEN mRNA expression levels in SKOV3/DDP cells transfected with the miRNA-21 inhibitors revealed no significant increase. The 
effect of miR-21 regulated the expression of PTEN mRNA was not clear. The upregulation of miRNA-21 in SKOV3 cells was concurrent with the downregulation of PTEN protein in these cells. The downregulation of miRNA-21 in SKOV3/DDP cells was also concurrent with the upregulation of PTEN protein in these cells. The present study demonstrated that miRNA-21 may have regulated cisplatin resistance by negatively targeting PTEN protein in EOC.

In conclusion, the present study demonstrated that miRNA-21 may have participated in cisplatin resistance in EOC. Furthermore, miRNA-21 may have regulated cisplatin resistance by negatively targeting PTEN protein in EOC. Future study should additionally consider cells with a higher resistance factor in order to further the study of cisplatin resistance. The association between the PTEN/PI3K/Akt signaling pathway, miRNA-21 and cisplatin resistance also requires further study.

\section{Acknowledgements}

The present study was supported by the National Natural Science Foundation of China (grant no. 81101973/H1621).

\section{References}

1. Vargas-Hernández VM, Moreno-Eutimio MA, Acosta-Altamirano G and Vargas-Aguilar VM: Management of recurrent epithelial ovarian cancer. Gland Surg 3: 198-202, 2014

2. Chen PS, Su JL and Hung MC: Dysregulation of microRNAs in cancer. J Biomed Sci 19: 90, 2012.

3. Garofalo M and Croce CM: MicroRNAs as therapeutic targets in chemoresistance. Drug Resist Updat 16: 47-59, 2013.

4. Song WF, Wang L, Huang WY, Cai X, Cui JJ and Wang LW: MiR-21 upregulation induced by promoter zone histone acetylation is associated with chemoresistance to gemcitabine and enhanced malignancy of pancreatic cancer cells. Asian Pac J Cancer Prev 14: 7529-7536, 2013.
5. Hong L, Han Y, Zhang Y, Zhang H, Zhao Q, Wu K and Fan D: MicroRNA-21: A therapeutic target for reversing drug resistance in cancer. Expert Opin Ther Targets 17: 1073-1080, 2013.

6. Wang ZX, LuBB, Wang H, Cheng ZX and Yin YM: MicroRNA-21 modulates chemosensitivity of breast cancer cells to doxorubicin by targeting PTEN. Arch Med Res 42: 281-290, 2011.

7. Yang SM, Huang C, Li XF, Yu MZ, He Y and Li J: miR-21 confers cisplatin resistance in gastric cancer cells by regulating PTEN. Toxicology 306: 162-168, 2013.

8. Komatsu S, Ichikawa D, Kawaguchi T, Miyamae M, Okajima W, Ohashi T, Imamura T, Kiuchi J, Konishi H, Shiozaki A, et al: Circulating miR-21 as an independent predictive biomarker for chemoresistance in esophageal squamous cell carcinoma. Am J Cancer Res 6: 1511-1523, 2016.

9. Shi GH, Ye DW, Yao XD, Zhang SL, Dai B, Zhang HL, Shen YJ, Zhu Y, Zhu YP, Xiao WJ and Ma CG: Involvement of microRNA-21 in mediating chemo-resistance to docetaxel in androgen-independent prostate cancer PC3 cells. Acta Pharmacol Sin 31: 867-873, 2010.

10. Milella M, Falcone I, Conciatori F, Cesta Incani U, Del Curatolo A, Inzerilli N, Nuzzo CM, Vaccaro V, Vari S, Cognetti F and Ciuffreda L: PTEN: Multiple Functions in Human Malignant Tumors. Front Oncol 5: 24, 2015.

11. Lou Y, Yang X, Wang F, Cui Z and Huang Y: MicroRNA-21 promotes the cell proliferation, invasion and migration abilities in ovarian epithelial carcinomas through inhibiting the expression of PTEN protein. Int J Mol Med 26: 819-827, 2010.

12. Mosmann T: Rapid colorimetric assay for cellular growth and survival: Application to proliferation and cytotoxicity assays. J Immunol Methods 65: 55-63, 1983.

13. Livak KJ and Schmittgen TD: Analysis of relative gene expression data using real-time quantitative PCR and the 2(-Delta Delta C(T)) Method. Methods 25: 402-408, 2001.

14. Li N, Yang L, Wang H, Yi T, Jia X, Chen C and Xu P: MiR-130a and MiR-374a Function as novel regulators of cisplatin resistance in human ovarian cancer A2780 Cells. PLoS One 10: e0128886, 2015.

15. Liu ZL, Wang H, Liu J and Wang ZX: MicroRNA-21 (miR-21) expression promotes growth, metastasis, and chemo- or radioresistance in non-small cell lung cancer cells by targeting PTEN. Mol Cell Biochem 372: 35-45, 2013. 